\section{P2-385 UNDERSTANDING PREVALENCE, MORBIDITIES AND FACTORS ASSOCIATED WITH OVERWEIGHT/OBESITY AMONG MARRIED WOMEN OF BHAKTAPUR CITY, NEPAL}

doi:10.1136/jech.2011.142976l.15

${ }^{1} \mathrm{~A}$ Devkota, ${ }^{*}{ }^{2,3} \mathrm{~B}$ Devkota, ${ }^{1} \mathrm{~K}$ Tiwari. ${ }^{1}$ Nepal Institute of Health Science, Purbanchal University, Kathmandu, Nepal; ${ }^{2}$ Tribhuvan University, Kathmandu, Nepal; ${ }^{3}$ Aberdeen University, Scotland, UK

Objective The aim of the study was to find out the prevalence, general morbidity patterns and the demographic/life style-related factors associated with overweight/obesity among women from different socio-economic groups residing in Bhaktapur City of Nepal.

Study design Cross-sectional descriptive.

Methods A total of 85 women, majority (53\%) comprising housewives, were recruited for structured interviews through a multistage random sampling method. Their height and weight were also measured during the interviews. The Body Mass Index (BMI) was calculated as per the WHO classification.

Results The study revealed that $44.7 \%$ women were overweight and $17.6 \%$ were obese. Overweight and obesity was higher in the age group 35 and above, non-breast feeding women and women with no or low mobility. Compared to non-obese women, the self- reported prevalence of asthma, low back pain and joint pain was statistically significantly higher among the overweight/obese women. Complaints of reproductive health problems like uterine and menstrual problems were also higher in overweight/obese women. The risk of low back pain among the overweight/obese women increased by 4.3 times compared to the underweight/normal group of women. The multiple binary regression analysis suggests that the lack of mobility or exercise is a main trigger for overweight/obesity among the married women in the study area.

Conclusion The prevalence of overweight/obesity among the married women was found to be higher than the national average for women. Hence targeted educational interventions are suggested to raise awareness about weight consciousness and healthy lifestyle choices among married women.

\section{P2-386 A META-ANALYSIS OF BUILT ROADWAY ENVIRONMENT AND PEDESTRIAN INJURY}

doi:10.1136/jech.2011.142976l.16

\section{DiMaggio. ${ }^{*}$ Columbia University, New York, New York, USA}

Changes to the built environment are an often-overlooked area of pedestrian injury control. To help address the need for baseline information to inform policy, we conducted a meta-analysis on the association of the roadway environment with the risk of pedestrian injury and mortality. We searched multiple electronic databases for studies related to built environment and pedestrian injuries. We abstracted and converted results to ORs, and synthesised the effect sizes with Bayesian meta analytic methods by placing vague or noninformative prior distributions for the central measure of effect and its variance on the likelihood of a set of normally distributed effect size logits. We identified 23 studies. Thirteen involved interventions, consisting of reconstruction (7), closures (4), and signage or unspecified (2). Ten studies were based on observational data. Studies were conducted in Europe (11), North America (4), Oceana (3), Asia (2), Africa (2), and South America (1). The overall association between roadway characteristics and risk of injury or death was $\mathrm{OR}=1.6$ (95\% CrI 1.2 to 2.1 ). There was an $80 \%$ probability that a future study would demonstrate a similar positive association. Roadway environment plays a crucial role in pedestrian injury and mortality. Public health and policy interventions to reduce the burden of such injuries should include attention to the built environment.

\section{P2-387 HEALTH PROFESSIONALS ARE NOT SYSTEMATIC IN OBESITY DETECTION AND MEASUREMENT}

doi:10.1136/jech.2011.142976l.17

${ }^{1} \mathrm{~K}$ Doherty, ${ }^{*}{ }^{1} \mathrm{~T}$ Ahern, ${ }^{1} \mathrm{~A}$ Billfalk-Kelly, ${ }^{1} \mathrm{D}$ Maeve, ${ }^{2} \mathrm{P}$ Fitzpatrick, ${ }^{1} \mathrm{I}$ Gilroy ${ }^{1} \mathrm{U}$ Mulholland, ${ }^{1} \mathrm{E}$ Rossiter, ${ }^{1,2} \mathrm{D}$ 0'Shea, ${ }^{1,2} \mathrm{C}$ Kelleher. ${ }^{1}$ St. Vincent's University Hospital, Dublin, Ireland; '2University College Dublin, Dublin, Ireland

Healthcare providers (HCPs) should identify overweight and obesity for surveillance and intervention purposes. This study aimed (a) to assess the ability of HCPs to estimate body mass index (BMI) by visual inspection and (b) to determine how systematically BMI is measured in an outpatient setting. A mixed group of 219 HCPs and 33 medical students were asked to estimate the BMI of five photographed people with varying degrees of obesity. A systematic survey of hospital charts took place over one week in a stratified random sample of 26 out-patient clinics in a teaching hospital. Each chart was surveyed for recording of height, weight, BMI and waist circumference at that clinic, or if not measured then, during the preceding year. The study group underestimated the BMI of obese people, with greater discrepancies as obesity increased: an actual BMI of $32 \mathrm{~kg} / \mathrm{m}^{2}$ was underestimated by $9 \%$, while one of $72 \mathrm{~kg} / \mathrm{m}^{2}$ was underestimated by $42 \%$. Of 515 hospital charts identified, weight was recorded for $33.4 \%$ of patients, height for $4 \%$, waist circumference for $1.4 \%$ and $\mathrm{BMI}$ for $0.4 \%$. In the 10 clinics that measured weight during the data collection period, the percentage of patients weighed was $11.8-88.9 \%$. However, clinics were reasonably well supplied with equipment: $100 \%$ had weighing scales, $76.9 \%$ had a measuring tape, $7.7 \%$ had a BMI chart and all but one had a stadiometer.

Conclusion HCPs neither recognise obesity reliably nor routinely record BMI. Systematic measurement strategies are required to tackle the global obesity epidemic.

\section{P2-388 CALCIUM IN BREAST MILK IS ASSOCIATED WITH THE UNDERWEIGHT OF INFANT AT AROUND 6 MONTHS AGE IN A COHORT OF BANGLADESH}

doi:10.1136/jech.2011.142976l.18

${ }^{1} \mathrm{M}$ Doi, ${ }^{2} \mathrm{R}$ S Rekha, ${ }^{2} \mathrm{~S}$ Ahmed, ${ }^{1} \mathrm{M}$ Okada, ${ }^{2} \mathrm{~A}$ K Roy, ${ }^{2} \mathrm{~S}$ E Arifeen, ${ }^{3} \mathrm{E}-\mathrm{C}$ Ekström, ${ }^{2} \mathrm{R}$ Raqib, ${ }^{1} \mathrm{Y}$ Wagatsuma. ${ }^{1}$ University of Tsukuba, Tsukuba, Japan; ${ }^{2}$ International Centre for Diarrhoeal Disease Research, Bangladesh (ICDDR,B), Dhaka, Bangladesh ${ }^{3}$ Uppsala University, Uppsala, Sweden

Introduction Appropriate growth of infants is important for their health and further life. In Bangladesh, there are still many infants below the growth standards. Calcium (Ca) is a major component of skeleton and it plays an important role in building bones early in life. The purpose of this study is to investigate the association between Ca level in breast milk and subsequent infants growth in rural Bangladesh

Methods The MINIMat ("Maternal and Infant Nutrition Interventions in Matlab") study was initiated by ICDDR,B. This study was nested into the overall MINIMat study. Breast milk samples at 2, 6 and 12 months of postpartum were acquired from 249 women. From obtained breast milk, Ca was measured. Infants' weight and length were attained at 2, 4, 6, 8, 12 and 15 months old and the zscores were calculated for indicators of infant growth.

Results Around $20 \%, 15 \%$ and $5 \%$ of this population was stunting, wasting and underweight. The multivariate regression analyses of $\mathrm{Ca}$ in serum and anthropometry indices showed that $\mathrm{Ca}$ was significantly associated with weight-for-height z-score (WHZ) and BMI-for-age $z$-score (BAZ) after adjusting for the effects of parity, wealth index, maternal weight, maternal age, birth weight and sex of the newborn ( 6 months, WHZ, $p=0.001$; BAZ, $p=0.001$; 8 months, WHZ, $p=0.001$; BAZ, $p=0.001)$. There was not significant relationship between other $\mathrm{z}$-score and $\mathrm{Ca}$ in breast milk. 